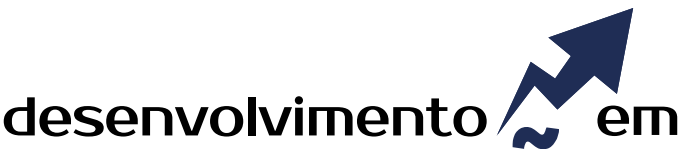 QUESTÃO
}

\section{Ranking de criação de valor das empresas sul-americanas Estudo Multicritério a Partir dos Métodos DP2 e VIKOR}

\author{
http://dx.doi.org/10.21527/2237-6453.2020.50.308-324
}

Recebido em: $14 / 1 / 2019$

Aceito em: 7/8/2019

\author{
Nelson Hein, ${ }^{1}$ Mara Vogt, ${ }^{2}$ Larissa Degenhart, ${ }^{3}$ \\ Darclê Costa Silva Haussmann, ${ }^{4}$ Adriana Kroenke ${ }^{5}$
}

\begin{abstract}
RESUMO
Este estudo objetivou analisar o ranking das empresas sul-americanas a partir dos métodos multicritério DP2 e VIKOR, considerando a criação de valor. Para tanto, foram analisadas as informações disponíveis de empresas de quatro países (Argentina, Brasil, Chile e Peru), correspondendo a um total de 69 empresas. Além disso, os dados foram referentes ao período de 2011 a 2015. Vale ressaltar que para mensurar a criação de valor optou-se pelas variáveis EVA e MVA. Com o intuito de elaborar o ranking de criação de valor para o EVA, MVA e EVA/MVA foram utilizados os métodos multicritério DP2 e VIKOR. A partir dos resultados conclui-se que as empresas dos quatro países sul-americanos analisados apresentaram posições muito próximas e em alguns casos, iguais nos dois rankings analisados (EVA e MVA). Sendo assim, ressalta-se que os métodos foram eficientes para identificar as empresas que mais criam valor nos países analisados, o que gera mais confiança por parte dos investidores, estes que podem considerar as duas variáveis de forma individual ou analisadas conjuntamente. A criação de valor é essencial para as empresas que buscam alavancar cada vez mais. A pesquisa torna-se relevante, pois as variáveis utilizadas para a realização deste estudo são essenciais, quando se trata de criação de valor em âmbito mundial. Além disso, contribui para que as empresas escolham as melhores opções de investimento, entre aquelas que apresentaram as informações necessárias, tendo em vista essa riqueza aqui exposta por meio de rankings.
\end{abstract}

Palavras-chave: Criação de valor. Métodos muticritério. DP2. VIKOR. América do Sul.

\section{RANKING OF VALUE CREATION OF SOUTH AMERICAN COMPANIES: MULTICRITERIA STUDY USING DP2 AND VIKOR METHODS}

\section{ABSTRACT}

This study aimed to analyze the ranking of South American companies from the multi-criteria DP2 and VIKOR, considering the creation of value. For this, the information available from companies from four countries (Argentina, Brazil, Chile and Peru) was analyzed, corresponding to a total of 69 companies. In addition, the data referred to the period from 2011 to 2015 . It is worth mentioning that in order to measure value creation, the variables EVA and MVA were chosen. In order to elaborate the value creation ranking for EVA, MVA and EVA / MVA, the multicriteria DP2 and VIKOR methods were used. From the results, it can be concluded that the companies of the four South American countries analyzed presented very close positions and, in some cases, were equal in the two analyzed rankings (EVA and MVA). Therefore, the methods were efficient to identify the companies that create the most value in the analyzed countries, which generates more confidence on the part of the investors, who can consider the two variables individually or, analyzed together. Value creation is essential for companies that are looking to leverage more and more. The research becomes relevant since the variables used to carry out this study are essential when it comes to creating value worldwide. In addition, it helps companies choose the best investment options among the companies that have submitted the necessary information, given the wealth presented here through rankings.

Keywords: Value creation. Methods muticriteria. DP2. VIKOR. South America.

\footnotetext{
${ }^{1}$ Doutor em Engenharia de Produção pela Universidade Federal de Santa Catarina (UFSC). Professor da Fundação Universidade Regional de Blumenau (Furb). hein@furb.br

${ }^{2}$ Doutoranda em Ciências Contábeis e Administração pela Fundação Universidade Regional de Blumenau (Furb). Bolsista Capes. maravogtcco@gmail.com

${ }^{3}$ Doutora em Ciências Contábeis pela Fundação Universidade Regional de Blumenau (Furb). Professora da Universidade Federal de Santa Maria (UFSM). lari_ipo@hotmail.com

${ }^{4}$ Doutoranda em Ciências Contábeis e Administração pela Fundação Universidade Regional de Blumenau (Furb). Professora da Fundação Universidade Regional de Blumenau (Furb).darcle@furb.br

${ }^{5}$ Doutora em Métodos Numéricos e Engenharia pela Universidade Federal do Paraná (UFPR). Professora da Fundação Universidade Regional de Blumenau (Furb). akroenke@furb.br
} 
Diante do crescimento acelerado no século 20, principalmente das grandes organizações, bem como com a entrada de mais acionistas no mercado acionário, gestores e proprietários apresentaram diferentes ideias, o que acabava gerando conflitos de agência, em que o gestor não se preocupava com a maximização da riqueza do acionista (SILVEIRA, 2002). A partir do desenvolvimento do mercado de capitais, contudo, houve um crescimento das empresas, fazendo com que aprimorassem as suas tecnologias e investissem cada vez mais para aumentar a produtividade (PAIVA; DE OLIVEIRA; PEIXOTO, 2015). Para mensurar essa riqueza, ou seja, a criação de valor, tem-se dois modelos mais utilizados e criados pelo economista Stern Stewart, o Economic Value Added (EVA), que é considerado o mais utilizado, e o Market Value Added (MVA) (BASSO; KRAUTER, 2003; BOURGUIGNON, 2005).

Nesse mesmo sentido, Bourguignon (2005) salienta que ambos, tanto o EVA quanto o MVA, encontram-se no cerne da criação de valor, entretanto fazem sentido apenas se forem tratados como elemento central da gestão financeira da organização. As duas ferramentas de criação de valor são consideradas as mais tradicionais e conhecidas (RAPPAPORT, 1998). Para Munteanu e Brezeanu (2012) o MVA reflete o valor excedente da organização, criado durante certo período de maior duração. Diante disso, haverá maior confiança por parte dos investidores em relação ao mercado de capitais e às perspectivas futuras dessas empresas que utilizam o MVA (MUNTEANU; BREZEANU, 2012).

De forma mais específica, tratando apenas do MVA, Alipour e Pejman (2015) destacam que este indicador se relaciona com a capacidade futura da empresa de criar valor aos investidores, sendo considerada uma medida externa. Tendo em vista a importância e necessidade de as empresas criarem valor, se isso não for possível é provável que elas encontrem dificuldades e tenham de enfrentar diversos desafios (KUMAR, 2016).

Diante do exposto e, levando em consideração que a discussão acerca da criação de valor não seja recente, não se localizou nenhum estudo que tenha analisado esta temática em empresas sul-americanas e que tenha verificado o ranking a partir de métodos multicritério, a fim de identificar as empresas que são mais atrativas para se investir, vislumbrando uma lacuna de pesquisa, além de contribuir para a literatura contábil por intermédio de um método que auxilia as tomadas de decisão. Desse modo, tem-se a seguinte questão que norteia esta pesquisa: Qual é o ranking das empresas sul-americanas a partir dos métodos multicritério DP2 e VIKOR, considerando a criação de valor? Nesse sentido, com o intuito de responder a essa questão, o objetivo deste estudo é analisar o ranking das empresas sul-americanas a partir dos métodos multicritério DP2 e VIKOR, considerando a criação de valor.

O estudo justifica-se, pois a criação de valor é de grande valia para as organizações, sejam estas pequenas ou grandes (LAURSEN; SVEJVIG, 2016), destacando-se que as técnicas EVA e MVA são importantes e confiáveis técnicas para mensurar esse valor, visando a atingir os objetivos dos proprietários, bem como dos acionistas, e são as técnicas mais comumente utilizadas pelas empresas, o que nos dias contemporâneos é cada vez mais relevante (ARAúJO; ASSAF NETO, 2003).

Além disso, os retornos do mercado de ações são primordiais para os acionistas, tendo em vista a busca constante por organizações mais atrativas e ao mesmo tempo confiáveis (WHEALE; AMIN, 2003). O fato de analisar as empresas sul-americanas é 
explicado por Costa, Lopes e De Oliveira Costa (2006), pois destacam que os países que compõem esse subcontinente da América encontram-se em crescimento, por mais que em alguns períodos este desenvolvimento não seja tão representativo.

A relevância do estudo e contribuição está pautada no fato de que a partir dos resultados, os interessados poderão analisar as organizações que apresentam maior criação de valor, bem como as tendências futuras a partir do período analisado. $O$ estudo também possibilitará a comparação entre as empresas do mesmo país, com os demais países e, ainda, a posição poderá ser confirmada devido à utilização de dois métodos diferentes, o que oferece maior confiança. Contribui também devido à utilização de métodos multicritério diferentes, justamente por auxiliar os gestores nas tomadas de decisão e por dar maior confiança em relação à classificação.

O estudo irá contribuir no que tange à concorrência das empresas, concorrência esta relacionada com a criação de valor, pois para Derfus et al. (2008), ela acaba dominando o mercado, fazendo com que este se desenvolva cada vez mais. Para evitar que isso aconteça é preciso que as empresas se esforcem para criar valor, inclusive a partir da introdução de novos produtos, métodos e iniciativas. Com vistas a alcançar o desempenho são necessárias diversas orientações estratégicas, determinantes para alcançar o resultado esperado (CADOGAN, 2012). Além disso, Tortella e Brusco (2003) frisam que medidas de criação de valor, como o EVA e MVA, servem de guia aos tomadores de decisões e como determinantes da remuneração dos executivos.

\section{CRIAÇÃO DE VALOR}

Uma empresa bem-sucedida não deverá ignorar as ações competitivas das demais organizações, mas sim, deverá agir de forma cada vez mais criativa e inovadora, sendo essa a principal fonte de expansão do mercado de ações e de crescimento da economia (SCHUMPETER, 1976). O objetivo da empresa, contudo, é ser um atrativo aos acionistas, a partir do momento em que cria valor (LUBATKIN; SHRIEVES, 1986). Vale ressaltar que a cultura organizacional contribui para a criação de valor e para o maior desempenho dos negócios (NARVER; SLATER, 1990).

John e Rue (1991) sugerem que os processos de planejamento auxiliam as organizações na criação de valor para se tornarem mais competitivas e para mudanças nas decisões tomadas, visando a um melhor desempenho de mercado. Estratégias são necessárias, pois a partir delas será possível desenvolver e explorar as vantagens competitivas, consideradas fontes de criação e valor, objetivo de qualquer empresa, seja esta grande ou pequena (BLACK; BOAL, 1994). Diante do exposto, entende-se que é necessário que as empresas usem sua criatividade, sejam inovadoras, empreendedoras, apresentem um bom planejamento e tenham boas estratégias para crescerem, se desenvolverem e competirem com as demais.

Além disso, é primordial que a organização se preocupe em criar valor. Para tanto, diversas são as medidas para verificar e mensurar a riqueza das empresas, uma das quais é o EVA, que foi desenvolvido por economistas há mais de 200 anos, como um sistema de gestão. Anos depois, após diversos estudos de Stern Stewart foi considerada uma estrutura para um sistema gerencial e financeiro, assim como para auxílio nas tomadas de decisão das empresas (GRANT, 1997). 
Vale destacar que de acordo com Rowe (2002), tanto em empresas novas quanto em empresas já estabelecidas a criação de valor é uma tarefa complexa e desafiadora, por meio da qual as empresas buscam alcançar a estabilidade financeira atual, bem como a viabilidade futura dos negócios. Assim, gestores acabam tomando decisões que maximizam ainda mais o retorno dos seus investimentos. Rowe (2002) ainda salienta que as empresas serão recompensadas com um desempenho superior no momento em que se preocuparem com a criação de valor.

A criação de valor busca pela interação entre as perspectivas operacional, financeira e estratégica, convergir o conhecimento individual em conhecimento organizacional, possibilitando respostas por meio de certos indicadores, gerados por um sistema de medição do desempenho (OLIVEIRA; BEUREN, 2003), como o EVA e MVA. Da mesma forma, Kayo et al., (2006) frisam que o conhecimento sobre a empresa pode contribuir para a adoção de estratégias para maximizar o seu valor econômico.

Por mais que a criação de valor tenha um conceito simples, ele é relevante e abrangente e há duas partes interessadas: o cliente e o acionista. Para criar valor ao cliente a empresa pode apresentar um produto valioso que ele esteja disposto a pagar. Já ao acionista a criação de valor é diferente (BOURGUIGNON, 2005), é a partir da vantagem competitiva que a organização apresenta. Assaf Neto (2003) acrescenta que a partir dessas medidas é possível obter vantagem competitiva e atrair os investidores.

Vale ressaltar que o conceito de criação de valor não é recente, visto que é tratado desde 1980 na obra do economista Alfred Marshall, intitulada "Capital, the principles of economics" (BURKSAITIENE, 2009). Especificamente o EVA foi conceituado como renda residual inicialmente, tem sido aperfeiçoado ao longo dos anos e renomeado pela empresa de consultoria Stern Stewart \& Co. como uma medida de gestão baseada em valor (ALIPOUR; PEJMAN, 2015). É, ainda, uma medida financeira considerada no mundo todo, que incentiva os gestores para agregarem e maximizarem valor aos seus interessados (BURKSAITIENE, 2009).

Já o MVA refere-se ao mercado e é considerado o valor de troca. A diferença obtida a partir desta medida indica a maximização de valor da empresa, referente a um longo período, o que fará com que a confiança dos investidores no mercado de capitais aumente ainda mais (MUNTEANU; BREZEANU, 2012). Para diferenciar o EVA do MVA, Berzakova, Bartosova e Kicova (2015) salientam que o MVA é o valor presente em relação aos demais valores utilizados para calcular o EVA, estes que serão obtidos no futuro.

Diante do contexto supracitado, Mietzner e Schiereck (2016) aduzem que, se os investidores estão interessados em auxiliar na gestão da empresa para criar valor, espera-se que haja maior reação do mercado perante estas empresas e, consequentemente, será possível que apresentem um maior potencial para crescimento.

\section{PROCEDIMENTOS METODOLÓGICOS}

Desenvolveu-se uma pesquisa descritiva, documental e quantitativa, com a finalidade de analisar o ranking das empresas sul-americanas por meio dos métodos multicritério DP2 e VIKOR, considerando a criação de valor. Diante disso, a população da pesquisa compreendeu todas as empresas de capital aberto deste subcontinente da América, totalizando 1.162 organizações. A amostra ficou limitada àquelas empresas que possuíam informações sobre todas as variáveis de desempenho de mercado e de criação de valor utilizadas. Sendo assim, a amostra constituiu-se de 69 empresas e ficou limitada a 4 países que possuíam as informações necessárias. Os países e quantidade de empresas é exposto a partir do Quadro 1. 
Quadro 1 - Amostra da pesquisa

\begin{tabular}{|c|c|c|}
\hline País & População & Amostra \\
\hline Argentina & 102 & 11 \\
\hline Brasil & 510 & 7 \\
\hline Chile & 253 & 35 \\
\hline Peru & 217 & 16 \\
\hline
\end{tabular}

Fonte: Base de dados Thomson (2016).

Ressalta-se que Paraguai e Uruguai não possuíam empresas na base de dados utilizada e Bolívia, Colômbia, Equador e Venezuela não apresentavam todas as informações necessárias para a realização do estudo, sendo assim eliminadas. Vale ressaltar que o período de análise foi de 2011 a 2015, ou seja, 5 anos, por conta de se tratar de países da América do Sul e no ano de 2011 ter sido constituída a Comunidade dos Estados Latino-Americanos e Caribenhos (Celac), com a finalidade do desenvolvimento econômico, entre outros objetivos (NÉSPOLI, 2013).

Em relação ao período de 5 anos de análise, Assaf Neto (2012) considera que é importante realizar uma análise temporal para acompanhar a evolução dos indicadores, em especial os de desempenho, o que deve ocorrer por um período de 3 a 5 anos. Quando se trata de vantagem competitiva, neste caso a partir da criação de valor, Powell (2003) defende que a apuração dos resultados necessita de um período adequado para o desenvolvimento de estratégias, o que pode variar de empresa para empresa e de setor para setor, contudo o autor observa que o período de 3 a 5 anos corresponde ao tempo médio da gestão e planejamento dos gestores (POWELL, 2003).

Sendo assim, neste estudo adotamos o período de 5 anos, iniciando em 2011 por conta da constituição da Celac nesse ano, conforme exposto anteriormente. Ressalta-se que outros estudos como o de Brito e Brito (2012) também utilizaram esse mesmo período para investigar a criação de valor. No Quadro 2 são evidenciadas as variáveis e os devidos autores.

Quadro 2 - Variáveis do estudo

\begin{tabular}{|c|c|c|c|}
\hline Variáveis & Definição & Fórmula & Autores \\
\hline $\begin{array}{l}\mathrm{EVA}^{\circ} \\
\text { (VEA) }\end{array}$ & $\begin{array}{c}\text { Economic } \\
\text { Value Added } \\
\text { (Valor Econô- } \\
\text { mico Agrega- } \\
\text { do) }\end{array}$ & $\begin{array}{l}\text { (ROI } \times \text { Investi- } \\
\text { mento) } \\
- \\
\text { (CMPC } \times \text { Investi- } \\
\text { mento) }\end{array}$ & $\begin{array}{c}\text { Grant (1997), Rappaport (1998), Araújo e Assaf } \\
\text { Neto (2003), Basso e Krauter (2003), Bourguig- } \\
\text { non (2005), Kayo et al. (2006), Beuren et al. } \\
\text { (2007), Assaf Neto, Lima e Araújo (2008), Burk- } \\
\text { saitiene (2009), Fiordelisi e Molyneux (2010), } \\
\text { Largani et al. (2012), Tortella e Brusco (2003) e } \\
\text { Alipour e Pejman (2015). }\end{array}$ \\
\hline $\mathrm{MVA}^{\circ}$ & $\begin{array}{l}\text { Valor de Mer- } \\
\text { cado Adicio- } \\
\text { nado (Market } \\
\text { Value Added) }\end{array}$ & 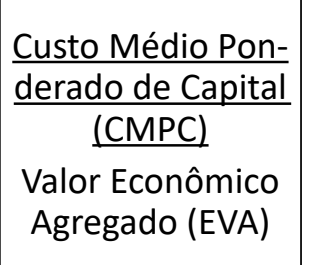 & $\begin{array}{c}\text { Grant (1997), Rappaport (1998), Araújo e Assaf } \\
\text { Neto (2003), Basso e Krauter (2003), Bourguig- } \\
\text { non (2005), Kayo et al. (2006), Beuren et al. } \\
\text { (2007), Burksaitiene (2009), Munteanu; Bre- } \\
\text { zeanu (2012), Largani et al. (2012) e Alipour e } \\
\text { Pejman (2015). }\end{array}$ \\
\hline
\end{tabular}

Fonte: Dados da pesquisa. 
Calculou-se o EVA a partir do proposto por Assaf Neto, Lima e Araújo (2008), conforme pode ser visualizado na Equação 1:

$$
\mathrm{EVA} \circledast \mathrm{B}=(\mathrm{ROI} \times \mathrm{I} \text { nvestimento })-(\mathrm{CMPC} \times \text { Investimento })
$$

Pode-se verificar que o ROI apresentado por meio da Equação 1, representa o Capital Investido e é obtido a partir da Equação 2:

$$
R O I=\left(\frac{\text { Lucro operacional-Impostos ou NOPAT) }}{\text { Investimento }}\right)
$$

Já o Custo Médio Ponderado de Capital (CMPC), também utilizado para cálculo do EVA, internacionalmente conhecido como Weighted Average Cost of Capital (WACC), é obtido a partir da Equação 3, apresentada por Assaf Neto, Lima e Araújo (2008):

$$
\begin{gathered}
C M P C=[(\% \text { Capital de Terceiros } \times \text { Custo do Capital de Terceiros }) \times(1-I R) \\
+(\text { Capital próprio } \times \text { Custo do Capital Próprio })]
\end{gathered}
$$

Para o cálculo do Custo do Capital de Terceiros (CCT), utilizado na fórmula anterior do CMPC, tem-se a Equação 4:

$$
C C T=\frac{\text { Total Despesas Financeiras }}{\text { Total Capital de Terceiros (empréstimos }+ \text { financiamento) }}
$$

Por fim, o Custo do Capital Próprio (CCP) também utilizado na fórmula do CMPC, é para Assaf Neto, Lima e Araújo (2008), mensurado conforme a Equação 5:

$$
C C P=R_{f}+\beta \times\left(R_{m}-R_{f}\right)
$$

Considera-se o $R_{f}$ como a taxa de retorno de um ativo livre de risco (Selic anual), o $\beta$ diz respeito ao coeficiente do título anual e foi obtido a partir da base de dados da Thomson ${ }^{\circ}$ e o $R_{m}$ representa a taxa de retorno anual de mercado. Subtraindo o $R_{f}$ pelo $R_{m}$ é possível obter o prêmio pelo risco de mercado (ASSAF NETO; LIMA; ARAÚJO, 2008).

A coleta de dados foi realizada por meio do banco de dados da Thomson ${ }^{\circ}$, na qual foram coletadas algumas das variáveis para cálculo da criação de valor. Já as variáveis Taxa Livre de Risco e a Taxa de Retorno de Mercado referem-se a cada país e foram retiradas do site do Banco Mundial. Para atender ao objetivo proposto neste estudo, o ranking de criação de valor foi elaborado a partir dos métodos DP2 e VIKOR, que são elucidados a seguir.

\section{Método DP2}

A Distância $\mathrm{P}_{2}$ é conhecida como método $\mathrm{DP}_{2}$ ou também, método DP2. Mais especificamente, é um modelo matemático utilizado para estimar indicadores de distância sintéticas e medir variáveis latentes (ZARZOSA ESPINA, 1996; JARAMILLO, 2008). O indicador sintético é um conceito de distância criado por Pena Trapero (1977) e indica que, devido ao fato de o resultado deste índice variar de acordo com a introdução de in- 
dicadores simples, o resultado final irá depender da variância de cada um destes (JARAMILLO, 2008). Para tanto, após Pena Trapero (1977) modificar essa distância obteve-se a seguinte fórmula, como pode ser visualizado a seguir:

$$
d_{I\left(i, i^{*}\right)}=\sum_{i=1}^{p} \frac{\left|x_{i j}-x_{i^{*} j}\right|}{\sigma_{i}} \prod_{i=1}^{j-1}\left(1-r_{i j, 1,2, \ldots, i-1}\right), \quad i<j
$$

No qual $r_{i j, 1,2, \ldots, i-1}$ é o coeficiente de correlação parcial entre o componente de ordem $i$ e $j$. Além disso, $d_{1}$ é uma medida que reflete o valor da diferença absoluta entre o conjunto de indicadores ideais $x_{i^{*} j}$ em relação a um conjunto de $p$ indicadores simples $x_{i j}$, tipificado pela inversão do desvio padrão do indicador simples, corrigindo informações redundantes a partir da inclusão do coeficiente de correlação parcial (JARAMILLO, 2008). O método DP2 de Pena Trapero (1977) foi baseado na distância de Frechet e utilizado como fator de ponderação das distâncias estimadas, ou seja, o coeficiente de determinação $\left(R^{2}\right)$ como segue:

$$
D P_{2}=\sum_{i=1}^{p} \frac{\left|x_{i j}-x_{i^{*} j}\right|}{\sigma_{i}}\left(1-R_{i, i-1, i-2, \ldots, 1}^{2}\right)
$$

Sendo assim, $d_{I}=\left|x_{i j}-x_{i^{*} j}\right|$ nos casos em que se tem indicadores de distância simples, como país, região, entre outros, em relação a um parâmetro de referência do indicador simples $X_{*}, \sigma_{i}$, o desvio padrão dos valores utiliza o indicador simples $i$ (JARAMILLO, 2008). O fator $\frac{d_{i}}{}$ consegue resolver o problema da heterogeneidade em relação às unidades de mediçăó dos indicadores simples, a fim de estabelecer a diferença estimada $\sigma_{i}$, o que assegura a propriedade aditiva em relação aos componentes do índice. Ainda, atua como fator de ponderação, dando maior importância às distâncias com valores mais elevados de dispersão ao comparar com a média (JARAMILLO, 2008).

Já o $R_{i, i-1, i-2, \ldots, 1}^{2}$ é considerado o coeficiente de determinação da regressão de $X_{i}$ na $X_{i-1} X_{i-2} \ldots X_{1}$, sendo este um número abstrato, que indica não haver importância em relação à unidade de medição. Além disso, tem-se o $R_{i}^{2}=0$ como primeiro componente que caso não haja componente anterior, apresenta todas as informações (JARAMILLO, 2008).

Para Jaramillo (2008), deve-se considerar que a ordem na qual os componentes são introduzidos altera o resultado final. O que diferencia o método DP2 dos demais métodos é o coeficiente de determinação que é utilizado e não a correlação. Diante disso, determina-se a hierarquia de componentes, visando a estimar o DP2, esta que se obtém por meio de um método da solução inicial. Por fim, o resultado geral dos indicadores simples indexados pelo DP2 é:

$$
D P_{2}=\frac{d_{1}}{\sigma_{1}}+\frac{d_{2}}{\sigma_{2}}\left(1-R_{2,1}^{2}\right)+\frac{d_{3}}{\sigma_{3}}\left(1-R_{3,2,1}^{2}\right)+\cdots+\frac{d_{p}}{\sigma_{p}}\left(1-R_{p, p-1, p-2, \ldots, 1}^{2}\right)
$$

Sendo assim, este método verifica a propriedade denominada de neutralidade e a distância $\mathrm{P}_{2}$ para satisfazer as condições de distância em determinado espaço métrico (Zarzosa Espina, 1996). 


\section{Método VIKOR}

O método ViseKriterijumska Optimizacija i Kompromisno Resenje (VIKOR) é também conhecido como Otimização Multicritério e Solução de Compromisso e compõe a Teoria Multiple Criteria Decision Making (MCDM), isto é, é um método multicritério utilizado para as tomadas de decisão (DUCKSTEIN; OPRICOVIC, 1980). O método assume relevância para a obtenção de rankings pois possibilita a otimização de sistemas complexos, auxiliando também na escolha de uma opção para cada função critério (TZENG; LIN; OPRICOVIC, 2005; OPRICOVIC; TZENG, 2007).

Este método tem como objetivo a criação de rankings, com alternativas de critérios diferentes e possibilita classificar as empresas o mais próximo do ideal (DUCKSTEIN; OPRICOVIC, 1980; OPRICOVIC; TZENG, 2004). Antes de obter o ranking deve-se seguir quatro etapas, as quais são apresentadas por Tzeng, Lin e Opricovic (2005) e Opricovic e Tzeng (2007):

Primeira etapa: Determinar os valores maiores $\left(f_{i}^{*}\right)$ e menores $\left(f_{i}^{-}\right)$de cada a função, $i=1,2, \ldots$, n. conforme exposto na Equação 1.

$$
f_{i}^{*}=\max _{j} f_{i j} \quad f_{i}^{-}=\min _{j} f_{i j}
$$

Onde:

$f_{i}^{*}$ : maior valor apresentado pelo indicador i conforme o conjunto de empresas;

$f_{i}^{-}$: menor valor apresentado pelo indicador i conforme o conjunto de empresas e $f_{i j}$ : o valor do indicador i atribuído à empresa $\mathrm{j}$.

Segunda etapa: Calcular os valores de $S_{j}$ (grupo de utilidade máxima) e de $R_{j}$ (peso individual mínimo), $j=1,2, \ldots, J$, nas relações, sendo $w_{i}$ os pesos de cada um dos critérios, obtidos a partir dos cálculos AHP. Tais cálculos são apresentados de acordo com as Equações 2 e 3.

$$
\begin{aligned}
& S_{j}=\frac{\sum_{i=1}^{n} w_{i}\left(f_{i}^{*}-f_{i j}\right)}{\left(f_{i}^{*}-f_{i}^{-}\right)} \\
& R_{j}=\max _{j}\left[\frac{w_{i}\left(f_{i}^{*}-f_{i j}\right)}{\left(f_{i}^{*}-f_{i}^{-}\right)}\right]
\end{aligned}
$$

Onde:

$S_{j}$ : grupo de utilidade máxima da empresa j;

$w_{i}$ : pesos dos critérios obtidos a partir do cálculo da entropia;

$f_{i}^{*}$ : maior valor evidenciado pelo indicador i atribuído pelo conjunto de empresas;

$f_{i j}$ : o valor do indicador i atribuído à empresa j;

$f_{i}^{-}$:menor valor apresentado pelo indicador i pelo conjunto de empresas e

$R_{j}$ : peso individual mínimo da empresa j. 
Na Figura 1 é possível verificar a área de compromissos, bem como de soluções ideais, tendo em vista os conjuntos viáveis e não viáveis, de acordo com os conjuntos obtidos a partir do cálculo.

Figura 1 - Área de compromissos e soluções ideais

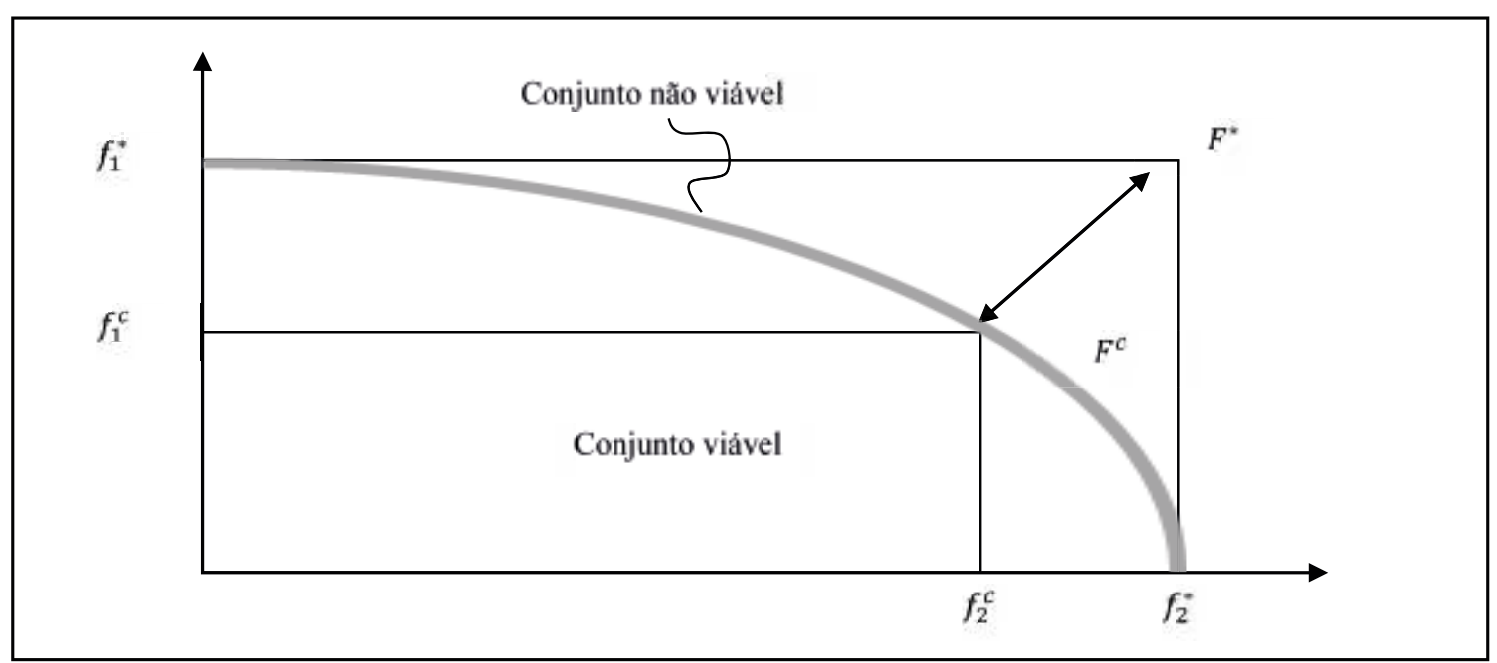

Fonte: OPRICOVIC; TZENG (2004).

Terceira etapa: Calcular os valores de $Q_{j}, j=1,2, \ldots, J$, a partir da relação, na qual $S^{*}=$ $\min _{j} S_{j}, S^{-}=\max _{j} S_{j}$ e $R^{*}=\min _{j} R_{j}, R^{-}=\max _{j} R_{j}$, o $v$ é incluído como peso de estratégia normalmente utilizado como $v=0,5$. Tal representação é exposta na Equação 4 que segue.

$$
Q_{j}=\frac{v\left(S_{j}-S^{*}\right)}{\left(S^{-}-S^{*}\right)}+\frac{(1-v)\left(R_{J}-R^{*}\right)}{\left(R^{-}-R^{*}\right)}
$$

Onde:

$v=0,5$;

$Q_{j}$ : score final da empresa j;

$S_{j}$ : grupo de utilidade máxima da empresa j;

$S^{*}$ : menor grupo de utilidade máxima do conjunto de empresas;

$S^{-}$: maior grupo de utilidade máxima do conjunto de empresas;

$R_{j}$ : peso individual mínimo;

$R^{*}:$ menor peso individual mínimo; e

$R^{-}$: maior peso individual mínimo. 
Quarta etapa: Classificar as alternativas em ordem decrescente, a partir dos valores obtidos por $\mathrm{S}, \mathrm{R}$ e $\mathrm{Q}$. Estes resultados geram três listas de classificação, contudo considera-se apenas os valores de $Q$.

Depois dos passos anteriormente apresentados, identifica-se o ranking de criação de valor para cada país. Na sequência é importante calcular o coeficiente de correlação de $\tau$ de Kendall, que trata de uma medida não paramétrica sobre o grau de correlação entre duas variáveis (X e Y) (KENDALL, 1970).

O autor ainda afirma que o coeficiente de Kendall não depende dos valores individuais das variáveis analisadas, mas de todos os rankings obtidos, observando-se que nesta pesquisa serão correlacionados os rankings de criação de valor obtidos por meio do método DP2 com os rankings anuais de criação de valor resultantes do método VIKOR, a fim de identificar se ambos apresentam resultados semelhantes, tornando-se assim, confiáveis. Para obter esse coeficiente utilizou-se o software Statistical Package for the Social Sciences (SPSS) ${ }^{\circledR}$.Destaca-se que tanto os rankings de criação de valor das empresas sul-americanas quanto a correlação, foram calculados individualmente para cada país, tendo em vista suas características próprias.

\section{ANÁLISE DOS DADOS}

A seguir são apresentados os resultados dos rankings de criação de valor, evidenciando na mesma tabela para cada país o ranking final do EVA e MVA, ou seja, da criação de valor, considerando as duas variáveis (EVA/MVA). Após, somente o ranking do EVA e, na 6a e 7a colunas o score e ranking do MVA. Vale ressaltar que o score diz respeito a todo o período analisado, isto é, de 2011 a 2015, a partir do método DP2 e na aequência, o método VIKOR. Inicialmente apresentam-se os resultados da Argentina, em seguida do Brasil, Chile e Peru, que foram os países que apresentavam todas as informações necessárias para o cálculo dos dois indicadores analisados. Na Tabela 1 evidencia-se o ranking de criação de valor das empresas argentinas. Destaca-se que foram apresentadas somente as 5 primeiras empresas de cada ranking para facilitar a visualização e comparação.

Tabela 1 - Rankings de criação de valor da Argentina

\begin{tabular}{|c|c|c|c|c|c|c|c|c|}
\hline Empresa & $\begin{array}{l}\text { Score } \\
\text { EVA/ } \\
\text { MVA }\end{array}$ & Ranking & $\begin{array}{c}\text { Score } \\
\text { EVA }\end{array}$ & Ranking & $\begin{array}{l}\text { Score } \\
\text { MVA }\end{array}$ & Ranking & Pts & $R k g$ \\
\hline \multicolumn{9}{|c|}{ Ranking DP2 } \\
\hline Con Del Oeste & 0,0168 & 1 & 0,0000 & 1 & 0,1848 & 1 & 30 & 1 \\
\hline Caputo SAIC & 0,0343 & 2 & 0,3669 & 2 & 0,2660 & 2 & 27 & 2 \\
\hline Grupo Clarin & 0,0873 & 3 & 0,4619 & 3 & 0,4894 & 3 & 24 & 3 \\
\hline Carboclor & 0,1303 & 4 & 1,0614 & 4 & 0,8591 & 4 & 21 & 4 \\
\hline Inst. Rosenbusch & 0,2300 & 6 & 1,2456 & 6 & 1,2902 & 5 & 16 & 5 \\
\hline \multicolumn{9}{|c|}{ Ranking VIKOR } \\
\hline Caputo SAIC & 0,0752 & 2 & 0,2590 & 2 & 0,0000 & 1 & 28 & 1 \\
\hline Con Del Oeste & 0,0000 & 1 & 0,3181 & 3 & 0,0310 & 2 & 27 & 2 \\
\hline Grupo Clarin & 0,1972 & 3 & 0,0000 & 1 & 0,1905 & 3 & 26 & 3 \\
\hline Carboclor & 0,4826 & 4 & 0,5786 & 4 & 0,4131 & 4 & 21 & 4 \\
\hline Inst. Rosenbusch & 0,6041 & 5 & 0,6068 & 5 & 0,5697 & 7 & 16 & 5 \\
\hline
\end{tabular}

Fonte: Dados da pesquisa. 
A partir dos dados apresentados na Tabela 1 nota-se que a empresa destaque em criação de valor no ranking pelo método DP2 é a Con Del Oeste, uma vez que foi a 1a colocada tanto para o EVA e MVA individual quanto quando analisados juntamente. Ressalta-se que não necessariamente o resultado deveria ser o mesmo, pois o MVA inclui em sua fórmula, além do EVA, o CMPC, também utilizado para o cálculo do EVA. Em todos os casos entende-se que nessa empresa (Cons Del Oeste), ambas as técnicas (EVA e MVA) são importantes e confiáveis para mensurar a criação de valor, conforme exposto na literatura por Araújo e Assaf Neto (2003) e servirão de guia aos tomadores de decisões (TORTELLA; BRUSCO, 2003).

Outro ponto que importa ser enfatizado é que somente a empresa Inst. Rosenbusch apresentou uma posição diferente de um ranking para o outro, mesmo que muito próximo, variando apenas uma posição. Os resultados demonstram que é possível e seguro confiar nessas empresas ao buscar identificar as que criam mais valor, pois mesmo mudando a variável analisada, apresentam boas posições.

Já no caso do método VIKOR percebe-se que as duas primeiras posições se inverteram ao comparar os resultados obtidos pelos dois métodos. Neste caso, a empresa que ocupou a 2a posição no ranking pelo método DP2 assumiu a liderança e a que assumia a liderança pelo método DP2 ficou na 2a posição. Em geral os resultados ficaram bem próximos, contudo houve mais oscilação no caso do VIKOR em relação às posições de uma variável para outra. Além disso, todas as 5 empresas que assumiram as primeiras posições são as mesmas nos dois rankings, o que é importante, até porque demonstra que ambos os métodos podem ser confiáveis. Isso pode ser confirmado a partir da Correlação $\tau$ de Kendall, que visa a identificar a relação dos rankings. Obteve-se um coeficiente de 0,891, que representa 89,1\%, isto é, uma forte correlação. Na Tabela 2 é possível visualizar os resultados dos rankings de criação de valor das empresas brasileiras.

Tabela 2 - Rankings de criação de valor do Brasil

\begin{tabular}{|lcccccc|c|c|}
\hline \multicolumn{1}{|c}{ Empresa } & $\begin{array}{c}\text { Score } \\
\text { EVA/ } \\
\text { MVA }\end{array}$ & Ranking & Score EVA Ranking & $\begin{array}{l}\text { Score } \\
\text { MVA }\end{array}$ & Ranking & Pts & Rkg \\
\hline Eletrobras & - & - & 0,0038 & $\mathbf{1}$ & 0,0088 & $\mathbf{1}$ & 19 & $\mathbf{1}$ \\
\hline Cyrela & - & - & 0,0669 & $\mathbf{2}$ & 0,4225 & $\mathbf{2}$ & 17 & $\mathbf{2}$ \\
\hline Whirlpool & - & - & 0,1371 & $\mathbf{5}$ & 0,6611 & $\mathbf{3}$ & 13 & $\mathbf{3}$ \\
\hline EZTEC & - & - & 0,1281 & $\mathbf{3}$ & 0,7298 & $\mathbf{7}$ & 11 & $\mathbf{4}$ \\
\hline Copasa & - & - & 0,1370 & $\mathbf{4}$ & 1,3059 & $\mathbf{7}$ & 10 & $\mathbf{5}$ \\
\hline Excelsior & - & - & 0,1742 & $\mathbf{6}$ & 1,0686 & $\mathbf{5}$ & 10 & $\mathbf{5}$ \\
\hline & & & Ranking VIKOR & & & & \\
\hline Eletrobras & 0,0000 & $\mathbf{1}$ & 0,0000 & $\mathbf{1}$ & 0,0000 & $\mathbf{1}$ & 18 & $\mathbf{1}$ \\
\hline Cyrela & 0,4201 & $\mathbf{2}$ & 0,4957 & $\mathbf{2}$ & 0,3603 & $\mathbf{2}$ & 15 & $\mathbf{2}$ \\
\hline EZTEC & 0,6871 & $\mathbf{3}$ & 0,7870 & $\mathbf{4}$ & 0,6548 & $\mathbf{4}$ & 10 & $\mathbf{3}$ \\
\hline Whirlpool & 0,7184 & $\mathbf{4}$ & 0,8160 & $\mathbf{5}$ & 0,6306 & $\mathbf{3}$ & 9 & $\mathbf{4}$ \\
\hline Excelsior & 0,8493 & $\mathbf{5}$ & 0,9007 & $\mathbf{6}$ & 0,7928 & $\mathbf{5}$ & 5 & $\mathbf{5}$ \\
\hline
\end{tabular}

Fonte: Dados da pesquisa. 
No caso das empresas brasileiras, ao se analisar os dois métodos é possível observar que a empresa que se destacou na 1a posição é a Eletrobras, no período de 2011 a 2015, tanto no ranking do EVA quanto no do MVA e em ambos os rankings. Na sequência tem-se a empresa Cyrela, que assumiu a mesma posição nos dois rankings, tanto no caso do EVA quanto do MVA. É possível notar que algumas empresas apresentaram posições distintas de uma variável para outra, porém não é representativo. Novamente, as 5 empresas que assumiram as primeiras posições de criação de valor são as mesmas nos dois rankings.

Sendo assim, a empresa Eletrobras, principalmente, demonstra que os investidores estão auxiliando na gestão para a criação de valor, o que vai ao encontro dos achados de Mietzner e Schiereck (2016), o que, ainda de acordo com os autores, possibilitará maior reação do mercado perante essas empresas. Além disso, corrobora os achados de Kayo et al. (2006) ao destacarem que a partir da criação de valor é possível que a empresa adote estratégias para maximizar ainda mais o seu valor econômico.

A partir da Correlação $\tau$ de Kendall verificou-se que o coeficiente foi de 0,293 e não significativo, o que demonstra que não houve correlação. Este fato, entretanto, pode ser explicado por serem poucas empresas analisadas, mas deve-se considerar seus resultados, tendo em vista que foram as empresas que apresentaram todas as informações necessárias nos anos analisados.

Vale ressaltar que devido ao fato de os valores do EVA e MVA dessas empresas serem altamente correlacionados, não foi possível calcular o ranking a partir do método DP2, pois o sistema trabalha matricialmente e não consegue inverter uma matriz singular (determinante0), isso por que o $\mathrm{R}^{2}$ foi igual 1 , o que inviabilizou o cálculo, tendo em vista que se necessita deste valor para cada um dos anos. A Tabela 3 apresenta o ranking de criação de valor das empresas chilenas.

Tabela 3 - Rankings de criação de valor do Chile

\begin{tabular}{|lccccccc|c|c|}
\hline \multicolumn{1}{|c}{ Empresa } & $\begin{array}{c}\text { Score } \\
\text { EVA/MVA }\end{array}$ & Ranking & $\begin{array}{c}\text { Score } \\
\text { EVA } \\
\text { Ranking DP2 }\end{array}$ & Ranking & $\begin{array}{c}\text { Score } \\
\text { MVA }\end{array}$ & Ranking & Pts & Rkg \\
\hline Falabella & 0,0673 & $\mathbf{1}$ & 0,1468 & $\mathbf{2}$ & 0,0339 & $\mathbf{1}$ & 101 & $\mathbf{1}$ \\
\hline Cemento Polpai & 0,3174 & $\mathbf{2}$ & 0,0093 & $\mathbf{1}$ & 0,5555 & $\mathbf{4}$ & 98 & $\mathbf{2}$ \\
\hline CTI & 0,4423 & $\mathbf{4}$ & 0,2895 & $\mathbf{6}$ & 0,4276 & $\mathbf{2}$ & 93 & $\mathbf{3}$ \\
\hline Quinenco & 0,4053 & $\mathbf{3}$ & 0,2248 & $\mathbf{5}$ & 0,5718 & $\mathbf{6}$ & 91 & $\mathbf{4}$ \\
\hline Elec Metalurgica & 0,4653 & $\mathbf{5}$ & 0,1589 & $\mathbf{3}$ & 0,7273 & $\mathbf{8}$ & 89 & $\mathbf{5}$ \\
\hline & \multicolumn{7}{c}{ Ranking VIKOR } \\
\hline Falabella & 0,0000 & $\mathbf{1}$ & 0,1041 & $\mathbf{2}$ & 0,0000 & $\mathbf{1}$ & 101 & $\mathbf{1}$ \\
\hline Cemento Polpai & 0,1058 & $\mathbf{2}$ & 0,0000 & $\mathbf{1}$ & 0,2201 & $\mathbf{2}$ & 100 & $\mathbf{2}$ \\
\hline CTI & 0,1841 & $\mathbf{3}$ & 0,1868 & $\mathbf{6}$ & 0,2370 & $\mathbf{3}$ & 93 & $\mathbf{3}$ \\
\hline Quinenco & 0,1890 & $\mathbf{4}$ & 0,1469 & $\mathbf{5}$ & 0,2714 & $\mathbf{4}$ & 92 & $\mathbf{4}$ \\
\hline Elec Metalurgica & 0,2009 & $\mathbf{5}$ & 0,1089 & $\mathbf{3}$ & 0,3083 & $\mathbf{8}$ & 89 & $\mathbf{5}$ \\
\hline
\end{tabular}

Fonte: Dados da pesquisa. 
A partir dos resultados expostos na Tabela 3 pode-se perceber que a empresa que assumiu a 1a posição nos rankings a partir dos dois métodos foi a mesma, ou seja, a empresa Falabella. De uma variável para a outra, contudo, a posição foi diferente. O que chama a atenção é que em ambos os métodos a posição em relação às variáveis foi a mesma. Novamente as empresas que ficaram entre as cinco primeiras posições são as mesmas e assumiram a mesma posição em cada um dos rankings.

Neste caso, tendo em vista que a empresa Falabella ficou em primeiro lugar nos dois rankings do MVA, pode-se apoiar nos argumentos de Munteanu e Brezeanu (2012) ao salientarem que esta variável reflete o valor excedente da organização, havendo maior confiança por parte dos investidores em relação ao mercado de capitais e às perspectivas futuras dessa empresa. No mesmo sentido, Alipour e Pejman (2015) corroboram os achados ao afirmar que o MVA se relaciona com a capacidade futura da empresa em criar valor aos investidores.

Para verificar a correlação dos dois rankings obtidos pelos métodos DP2 e VIKOR, calculou-se a Correlação $\tau$ de Kendall, sendo o coeficiente de correlação de 0,953 e significativo, ou seja, os rankings apresentam 95,3\% de correlação, o que é considerado uma forte correlação. É notável que algumas empresas apresentaram posições distintas de uma variável para a outra. Essa diferença pode ser explicada pelo fato de o MVA ser composto além da variável EVA, pelo CMPC. Também destaca-se que o fato de serem mais empresas que compõem a amostra deste país, pode explicar o motivo de elas não apresentarem sempre a mesma posição nas variáveis analisadas. Na Tabela 4 é exposto o ranking de criação de valor das empresas peruanas.

Tabela 4 - Rankings de criação de valor do Peru

\begin{tabular}{|lccccccc|c|c|}
\hline \multicolumn{1}{|c}{ Empresa } & $\begin{array}{c}\text { Score } \\
\text { EVA/MVA }\end{array}$ & $\begin{array}{c}\text { Ranking } \\
\text { Ranking DP2 } \\
\text { EVA }\end{array}$ & $\begin{array}{c}\text { Score } \\
\text { Ranking }\end{array}$ & $\begin{array}{c}\text { Score } \\
\text { MVA }\end{array}$ Ranking & Pts & Rkg \\
\hline Telefonica & 0,1927 & $\mathbf{1}$ & 0,0000 & $\mathbf{1}$ & 0,6076 & $\mathbf{1}$ & 45 & 1 \\
\hline Backus Johnston & 0,2708 & $\mathbf{2}$ & 0,1969 & $\mathbf{2}$ & 0,6288 & $\mathbf{2}$ & 42 & 2 \\
\hline Lima Caucho & 0,4926 & $\mathbf{3}$ & 0,5731 & $\mathbf{3}$ & 1,7409 & $\mathbf{3}$ & 39 & 3 \\
\hline Falabella Peru & 0,7163 & $\mathbf{5}$ & 0,6242 & $\mathbf{4}$ & 2,1358 & $\mathbf{5}$ & 34 & 4 \\
\hline Alicorp & 0,6589 & $\mathbf{4}$ & 0,8097 & $\mathbf{5}$ & 2,1893 & $\mathbf{6}$ & 33 & 5 \\
\hline & \multicolumn{7}{c}{ Ranking VIKOR } \\
\hline Backus Johnston & 0,0000 & $\mathbf{1}$ & 0,0431 & $\mathbf{2}$ & 0,0000 & $\mathbf{1}$ & 44 & $\mathbf{1}$ \\
\hline Telefonica & 0,1064 & $\mathbf{2}$ & 0,0000 & $\mathbf{1}$ & 0,1651 & $\mathbf{2}$ & 43 & $\mathbf{2}$ \\
\hline Lima Caucho & 0,2814 & $\mathbf{3}$ & 0,2771 & $\mathbf{3}$ & 0,3179 & $\mathbf{3}$ & 39 & $\mathbf{3}$ \\
\hline Falabella Peru & 0,3685 & $\mathbf{4}$ & 0,3209 & $\mathbf{4}$ & 0,4095 & $\mathbf{4}$ & 36 & $\mathbf{4}$ \\
\hline Cia Minera Milp & 0,4611 & $\mathbf{5}$ & 0,5684 & $\mathbf{6}$ & 0,4313 & $\mathbf{5}$ & 32 & $\mathbf{5}$ \\
\hline
\end{tabular}

Fonte: Dados da pesquisa.

Em relação aos resultados dos rankings das empresas peruanas evidenciados pela Tabela 4, destaca-se que a empresa Telefonica a partir do método DP2 assumiu a 1a posição nas três variáveis analisadas, ou seja, EVA/MVA, EVA e MVA. Já a partir do método VIKOR, a sua posição final foi como 2aㅡ, porém na variável EVA ficou com a 1a posição. Diante disso, este achado corrobora o resultado encontrado por Burksaitiene (2009), ao também verificar que especificamente o EVA incentiva os gestores para agregarem e 
maximizarem valor aos seus interessados. Além dessa empresa, vale destacar a Backus Johnston, que liderou o ranking pelo método VIKOR e foi a $2^{\text {a }}$ colocada no ranking pelo método DP2.

O fato, entretanto, de as duas empresas (Telefonica e Backus Johnston) terem ficado bem colocadas em ambos os rankings (DP2 e VIKOR), demonstra que essas são duas ótimas opções para os investidores que buscam por empresas que criam valor, levando em consideração, neste caso, o EVA e MVA.

Da mesma forma que nos demais países analisados por meio deste estudo, é possível notar que a maioria das empresas apresentou variação de um ranking para o outro. Em relação a correlação $\tau$ de Kendall, enfatiza-se que houve uma forte correlação de 0,883 e, significativa, o que denota que é possível confiar em ambos os métodos utilizados, ou seja, DP2 e VIKOR.

\section{CONSIDERAÇÕES FINAIS}

O estudo teve por objetivo analisar o ranking das empresas sul-americanas a partir dos métodos multicritério DP2 e VIKOR, considerando a criação de valor em uma amostra de 69 empresas, que dizem respeito aos países: Argentina, Brasil, Chile e Peru, que apresentavam todas as informações necessárias para a realização deste estudo, no período de 2011 a 2015. Destaca-se que para a criação de valor considerou-se os indicadores EVA e MVA.

Os achados revelaram que os dois métodos apresentaram resultados muito próximos em relação ao ranking das empresas dos quatro países, sendo as posições finais muitas vezes as mesmas, contudo em alguns países mudava uma posição, o que pode ser explicado devido ao fato de os métodos serem operacionalizados de formas distintas, levando a alterar, mesmo que de forma sutil, o ranking.

Nesse sentido, por mais que tenham sido expostos os escores e posições das cinco primeiras empresas classificadas de cada país analisado, a correlação $\tau$ de Kendall foi em relação a todas as empresas de cada país, o que confirma esta classificação próxima entre os dois métodos utilizados, ou seja, DP2 e VIKOR.

Verificou-se que apenas no caso das empresas brasileiras não houve correlação significativa, o que pode ser explicado pelo fato de terem sido poucas empresas analisadas. Nos demais países, porém, a correlação foi significativa e alta, o que é muito importante, pois pode-se inferir que os métodos DP2 e VIKOR são eficientes para análise da criação de valor.

A partir dos resultados e com vistas a responder ao problema de pesquisa e atingir o objetivo proposto de analisar o ranking das empresas sul-americanas a partir dos métodos multicritério DP2 e VIKOR, considerando a criação de valor, conclui-se que as empresas dos quatro países sul-americanos estudados apresentaram posições muito próximas e em alguns casos iguais nos dois rankings analisados. Sendo assim, ressalta-se que os métodos foram eficientes para identificar as empresas que mais criam valor nesses países, o que gera mais confiança por parte dos investidores, os quais podem considerar as duas variáveis de forma individual ou analisadas conjuntamente. 
A criação de valor é essencial para as empresas que buscam crescer cada vez mais. Burksaitiene (2009) salienta que é importante que se utilize a criação de valor para monitorar o valor econômico das organizações. Além disso, Tortella e Brusco (2003) complementam que essas informações podem auxiliar os gestores nas tomadas de decisão no que tange aos investimentos momentâneos, bem como futuros, servindo também para determinar a remuneração dos seus executivos.

Mesmo que tenham sido poucas empresas analisadas, em comparação com o total de cada país, a pesquisa torna-se relevante, pois as variáveis utilizadas para a realização deste estudo são essenciais, quando se trata de criação de valor em âmbito mundial. Além disso, contribui para que as empresas escolham as melhores opções de investimento, entre as empresas que apresentaram as informações necessárias, tendo em vista essa riqueza aqui exposta por meio de rankings. Diante do exposto, Kumar (2016) frisa que a partir dos resultados os acionistas terão condições para avaliar as economias com maiores retornos, o que minimizará a assimetria informacional presente no mercado. No que diz respeito aos métodos multicritérios, esta pesquisa contribui, pois eles auxiliam os gestores nas tomadas de decisão, apontando os melhores caminhos a serem seguidos.

Levando em consideração as limitações do estudo, como a quantidade de países e empresas que apresentavam as informações necessárias para compor as variáveis analisadas, ou seja, EVA e MVA, enfatiza-se que os resultados não devem ser generalizados. Outra limitação diz respeito ao número de variáveis, o período e até mesmo os métodos multicritério utilizados. Sendo assim, sugere-se que os estudos futuros levem em consideração mais variáveis para mensurar a criação de valor, a utilização de outros métodos multicritério para fins de comparação dos resultados e o período analisado, o qual pode alterar os resultados obtidos, tendo em vista as mudanças na economia, bem como a análise de outros países.

\section{REFERÊNCIAS}

ALIPOUR, Mohammad; PEJMAN, Mohammad Ebrahim. The impact of performance measures, leverage and efficiency on market value added: Evidence from Iran. Global Economics and Management Review, v. 20, n. 1, p. 6-14, 2015.

ARAÚJO, Adriana Maria Procópio de; ASSAF NETO, Alexandre. A contabilidade tradicional e a contabilidade baseada em valor. Revista Contabilidade \& Finanças, v. 14, n. 33, p. 16-32, 2003.

ASSAF NETO, Alexandre. Contribuição ao estudo de avaliação de empresas no Brasil: uma aplicação prática. 2003. 203f. Tese (Livre-Docência) - Universidade de São Paulo, Faculdade de Economia, Administração e Contabilidade, Ribeirão Preto, 2003.

ASSAF NETO, Alexandre. Finanças corporativas e valor. 6. ed. São Paulo: Atlas, 2012.

ASSAF NETO, Alexandre; LIMA, Fabiano Guasti; ARAÚJO, Adriana Maria Procópio de. Uma proposta metodológica para o cálculo do custo de capital no Brasil. Revista de Administração-Rausp, v. 43, n. 1, p. 72-83, 2008.

BASSO, Leonardo Fernando Cruz; KRAUTER, Elizabeth. Participação nos lucros/resultados e criação de valor: um estudo exploratório. Organizações \& Sociedade, v. 10, n. 26, p. 157-178, 2003.

BERZAKOVA, Viera; BARTOSOVA, Viera; KICOVA, Eva. Modification of EVA in value based management. Procedia Economics and Finance, v. 26, p. 317-324, 2015.

BEUREN, Ilse Maria; HEIN, Nelson; CAVASSOLA, Jerber Antonio. Participação de Mercado e Criação de Valor: um Estudo de Empresas do Varejo Automobilístico em Santa Catarina. RAC-Eletrônica, v. 1, n. 3, p. 14-34, 2007.

BLACK, Janice A.; BOAL, Kimberly B. Strategic resources: Traits, configurations and paths to sustainable competitive advantage. Strategic Management Journal, v. 15, n. S2, p. 131-148, 1994. 
BOURGUIGNON, Annick. Management accounting and value creation: the profit and loss of reification. Critical Perspectives on Accounting, v. 16, n. 4, p. 353-389, 2005.

BRITO, Renata Peregrino de; BRITO, Luiz Artur Ledur. Vantagem competitiva, criação de valor e seus efeitos sobre o desempenho. RAE-Revista de Administração de Empresas, v. 52, n. 1, p. 70-84, 2012.

BURKSAITIENE, Daiva. Measurement of value creation: Economic value added and net present value. Economics and Management, n. 14, p. 709-714, 2009.

CADOGAN, John W. International marketing, strategic orientations and business success: Reflections on the path ahead. International Marketing Review, v. 29, n. 4, p. 340-348, 2012.

COSTA, Fábio Moraes; LOPES, Alexsandro Broedel; DE OLIVEIRA COSTA, Alessandra Cristina. Conservadorismo em cinco países da América do Sul. Revista Contabilidade \& Finanças, v. 17, n. 41, p. 7-20, 2006.

DUCKSTEIN, Lucien; OPRICOVIC, Serafim. Multiobjective optimization in river basin development. Water Resources Research, v. 16, n. 1, p. 14-20, 1980.

DERFUS, P. J.; MAGGITTI, P. G.; GRIMM, C. M.; SMITH. The Red Queen effect: Competitive actions and firm performance. Academy of Management Journal, v. 51, n. 1, p. 61-80, 2008.

FIORDELISI, Franco; MOLYNEUX, Phil. The determinants of shareholder value in European banking. Journal of Banking \& Finance, v. 34, n. 6, p. 1.189-1.200, 2010.

GRANT, J. L. Foundations of Economic Value Added. New Hope, Pennsylvania: By Frank J. Fabozzi Associates, 1997.

JARAMILLO, Luis Alfonso Escobar. Indicadores ambientales sintéticos: una aproximación conceptual desde la estadística multivariante. Gestión y Ambiente, v. 11, n. 1, p. 121-140, 2008.

JOHN, Caron H.; RUE, Leslie W. Research notes and communications co-ordinating mechanisms, consensus between marketing and manufacturing groups, and marketplace performance. Strategic Management Journal, v. 12, n. 7, p. 549-555, 1991.

KAYO, E. K.; KIMURA, H.; MARTIN, D. M. L.; NAKAMURA, W. T. Ativos intangíveis, ciclo de vida e criação de valor. Revista de Administração Contemporânea, v. 10, n. 3, p. 73-90, 2006.

KENDALL, M. G. Rank Correlation Methods. London: Charles Griffin \& Co, 1970.

KUMAR, R. Valuation: Theories and Concepts - Perspectives on value and valuation. 2016. Available from: http://www.sciencedirect.com/science/book/9780128023037. Cited: 20 Nov. 2018.

LARGANI, Mahmoud Samadi; KAVIANI, Meysam; ABDOLLAHPOUR, Amirreza. A review of the application of the concept of Shareholder Value Added (SVA) in financial decisions. Procedia-Social and Behavioral Sciences, v. 40, p. 490-497, 2012.

LAURSEN, Markus; SVEJVIG, Per. Taking stock of project value creation: A structured literature review with future directions for research and practice. International Journal of Project Management, v. 34, n. 4, p. 736-747, 2016.

LUBATKIN, Michael; SHRIEVES, Ronald E. Towards reconciliation of market performance measures to strategic management research. Academy of Management Review, v. 11, n. 3, p. 497-512, 1986.

MIETZNER, Mark; SCHIERECK, Dirk. Value creation by block acquisitions and the importance of block owner identity. Finance Research Letters, v. 17, p. 118-124, 2016.

MUNTEANU, Anca; BREZEANU, Petre. Do Romanian Banking Institutions Create Shareholder Value? Procedia Economics and Finance, v. 3, p. 144-151, 2012.

NARVER, John C.; SLATER, Stanley F. The effect of a market orientation on business profitability. The Journal of marketing, v. 54, n. 4, p. 20-35, 1990.

NÉSPOLI, Gabriela. Conheça oito fatos marcantes sobre integração regional na América Latina. 2013. Disponível em: https://operamundi.uol.com.br/politica-e-economia/32669/conheca-oito-fatos-marcantes-sobre-integracao-regional-na-america-latina. Acesso em: 9 jul. 2019.

OLIVEIRA, Joel Marques de; BEUREN, Ilse Maria. O tratamento contábil do capital intelectual em empresas com valor de mercado superior ao valor contábil. Revista Contabilidade \& Finanças, v. 14, n. 32, p. 81-98, 2003.

OPRICOVIC, Serafim; TZENG, Gwo-Hshiung. Extended VIKOR method in comparison with outranking methods. European Journal of Operational Research, v. 178, n. 2, p. 514-529, 2007.

OPRICOVIC, Serafim; TZENG, Gwo-Hshiung. Compromise solution by MCDM methods: A comparative analysis of VIKOR and TOPSIS. European Journal of Operational Research, v. 156, n. 2, p. 445-455, 2004.

PAIVA, José Fernando Martins; DE OLIVEIRA, Nelize Aparecida; PEIXOTO, Fernanda Maciel. A relação entre conselho de administração, desempenho, valor e risco no mercado brasileiro de ações. Revista de Educação e Pesquisa em Contabilidade (REPeC), v. 9, n. 1, p. 25-44, 2015. 
PENA TRAPERO, J. B. Problemas de la medición del bienestar y conceptos afines: una aplicación al caso español. Madrid: INE, 1977.

POWELL, Thomas C. Varieties of competitive parity. Strategic Management Journal, v. 24, n. 1, p. 61-86, 2003.

RAPPAPORT, A. Creating Shareholder Value. 2. ed., New York: The Free Press, 1998.

ROWE, W. Glenn. Liderança estratégica e criação de valor. Revista de Administração de Empresas, v. 42, n. 1, p. 1-15, 2002.

SCHUMPETER, J. A. Capitalism, Socialism, and Democracy. 5. ed. London: George Allen \& Unwin, 1976.

SILVEIRA, A. D. M. Governança corporativa, desempenho e valor da empresa no Brasil. 2002. 165f. Dissertação (Mestrado em Administração) - Universidade de São Paulo, Faculdade de Economia, Administração e Contabilidade, 2002.

TORTELLA, Bartolomé Deyá; BRUSCO, Sandro. The Economic Value Added (EVA): an analysis of market reaction. Advances in Accounting, v. 20, p. 265-290, 2003.

TZENG, Gwo-Hshiung; LIN, Cheng-Wei; OPRICOVIC, Serafim. Multi-criteria analysis of alternative-fuel buses for public transportation. Energy Policy, v. 33, n. 11, p. 1.373-1.383, 2005.

ZARZOSA ESPINA, M. Del Pilar. Aproximación a la medición del bienestar social. Idoeneidad del indicar sintético “Distancia-P (2)". (Aplicación al caso español). Cuadernos de Economía, v. 24, n. 68, p. 139-163, 1996.

WHEALE, Peter Robert; AMIN, Laura Heredia. Bursting the dot. com "Bubble": A case study in investor behaviour. Technology Analysis \& Strategic Management, v. 15, n. 1, p. 117-136, 2003. 